\title{
Liposarcoma arising from the right renal sinus with inferior vena caval involvement
}

\author{
Yasuyuki Kobayashi ${ }^{1}$, Masashi Nakayama ${ }^{1}$, Kyosuke Matsuzaki $^{1}$, Ken Takeda ${ }^{1}$, Takahiro \\ Yoshida', Yasuyuki Arai ${ }^{1}$, Kenichi Kakimoto', Michiko Yuki ${ }^{2}$, Yasuhiko Tomita², Yutaka Ono ${ }^{3}$, \\ Kazuo Nishimura ${ }^{1}$ \\ 1. Department of Urology, Osaka Medical Center for Cancer and Cardiovascular Diseases, Osaka, Japan. \\ 2. Department of Pathology, Osaka Medical Center for Cancer and Cardiovascular Diseases, Osaka, Japan. \\ 3. Department of Urology, Higashiosaka City General Hospital, Higashiosaka, Japan \\ Correspondence: Yasuyuki Kobayashi. Address: Department of Urology, Osaka Medical Center for Cancer and \\ Cardiovascular Diseases, 1-3-3 Nakamichi Higashinari, Osaka 537-8511, Japan. \\ Email: ya_su_koba@yahoo.co.jp
}

Received: March 12, 2015

Accepted: June 2, $2015 \quad$ Online Published: June 8, 2015

DOI : $10.5430 /$ jst.v5n2p69

URL: http://dx.doi.org/10.5430/jst.v5n2p69

\begin{abstract}
A woman aged 55 was introduced to our medical center for retroperitoneal tumor incidentally discovered by screening computerized tomography. Imaging showed a round fatty tumor extending into the right kidney and infiltrating into the inferior vena cava and no distant metastasis. We extirpated the tumor and tumor thrombus infiltrating the inferior vena cava along with the right kidney en bloc. Pathological examination showed well-differentiated liposarcoma that we believe had arisen from the renal sinus. Liposarcoma arising from the renal sinus with intravenous involvement is distinctly rare. Treatment by complete resection is recommended.
\end{abstract}

\section{Key words}

Liposarcoma, Renal sinus, Intravenous

\section{I ntroduction}

Primary retroperitoneal tumors are reported to account for $0.16 \%$ of all malignancies, and $10 \%-20 \%$ of all primary retroperitoneal tumors are liposarcomas ${ }^{[1]}$. Although liposarcoma is the second most common type of renal sarcoma ${ }^{[2]}$, renal liposarcoma with inferior vena caval involvement is distinctly rare. To our knowledge, this is only the third reported case of renal liposarcoma.

\section{Case report}

A woman aged 55 was introduced to our medical center for retroperitoneal tumor incidentally discovered by screening computerized tomography (CT) during a pretreatment work-up for early breast cancer. She had no history of urologic disease. Urinalysis and blood test results were normal. Physical findings were unremarkable. CT showed an elliptic fatty tumor $5 \mathrm{~cm} \times 7 \mathrm{~cm}$ in diameter in the right renal hilus (see Figure 1a). The tumor infiltrated into the right kidney and right 
renal vein (see Figure 1b). Magnetic resonance imaging (MRI) also showed a tumor infiltrating into the right kidney and tumor thrombus involving the inferior vena cava (IVC) (see Figure 2).

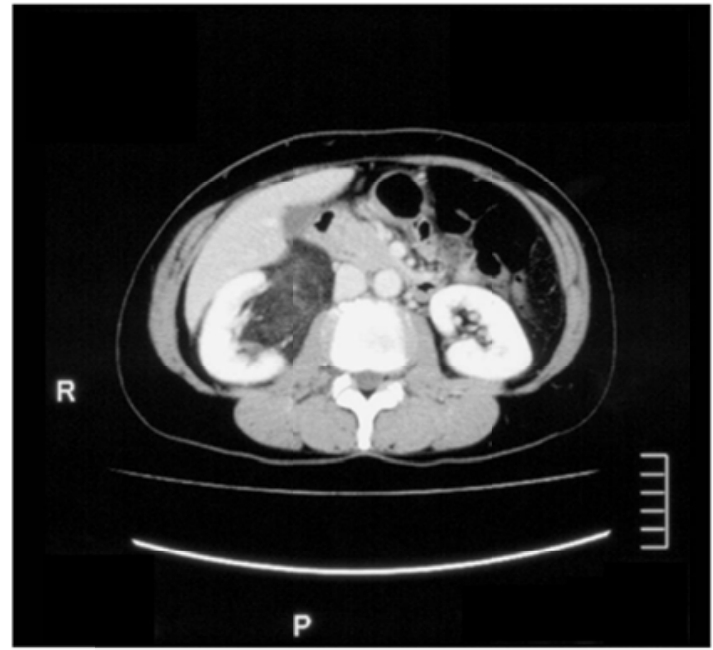

a

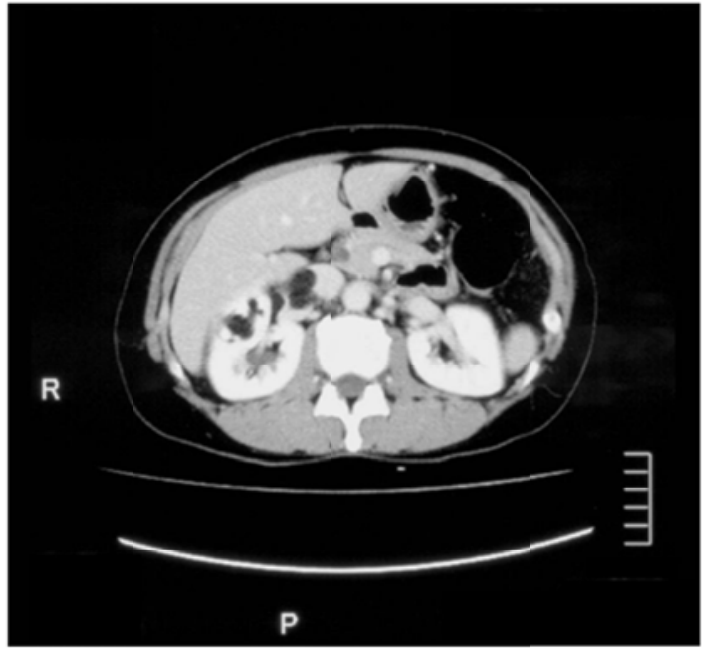

b

Figure 1. Abdominal computerized tomography showed an elliptic fatty tumor in the right renal hilus (a), which was expanding into the right kidney (arrow) and the right renal vein (arrowhead) (b)

Figure 2. Magnetic resonance imaging showed a tumor with thrombus extending to the inferior border of the liver (arrow)

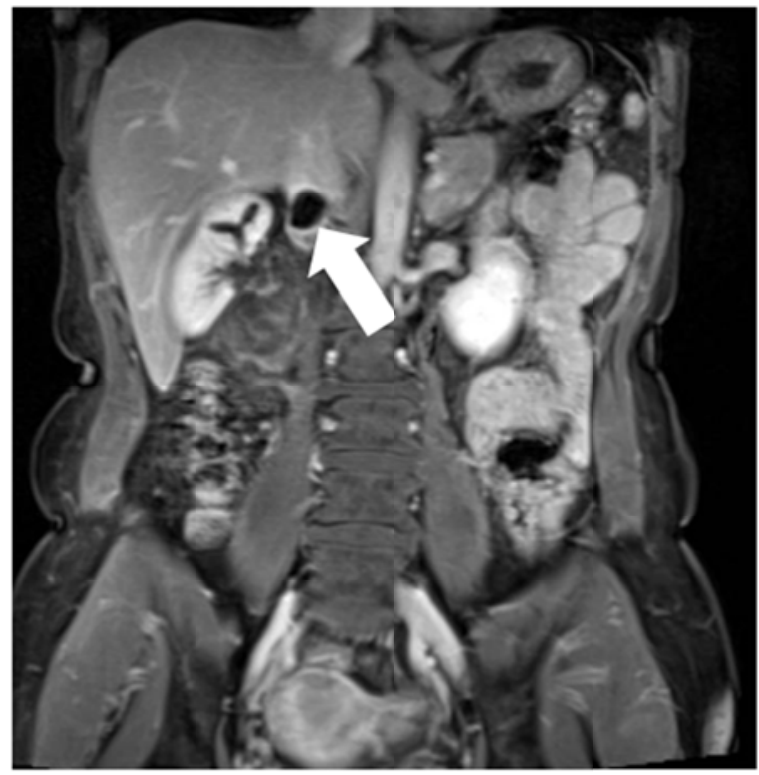

We surgically removed the tumor and tumor thrombus together with the right kidney. On gross examination, the deep yellow tumor infiltrated into the renal parenchyma (see Figure 3) and extended into the right renal vein. The tumor consists of mature fat with a variable number of spindled cells with hyperchromatic nuclei and multivacuolated lipoblasts (see Figure 4a). The pathologic examination showed well-differentiated liposarcoma, which we believe had arisen from within the renal sinus. The tumor infiltrated into renal parenchyma (see Figure 4b). Histological examination showed that the tumor had infiltrated into the renal vein (see Figure 4c) and IVC. The patient has had no recurrence of tumor during a follow-up period of 8 months. 
Figure 3. Macroscopic appearance showed a tumor arising from renal sinus adipose tissue
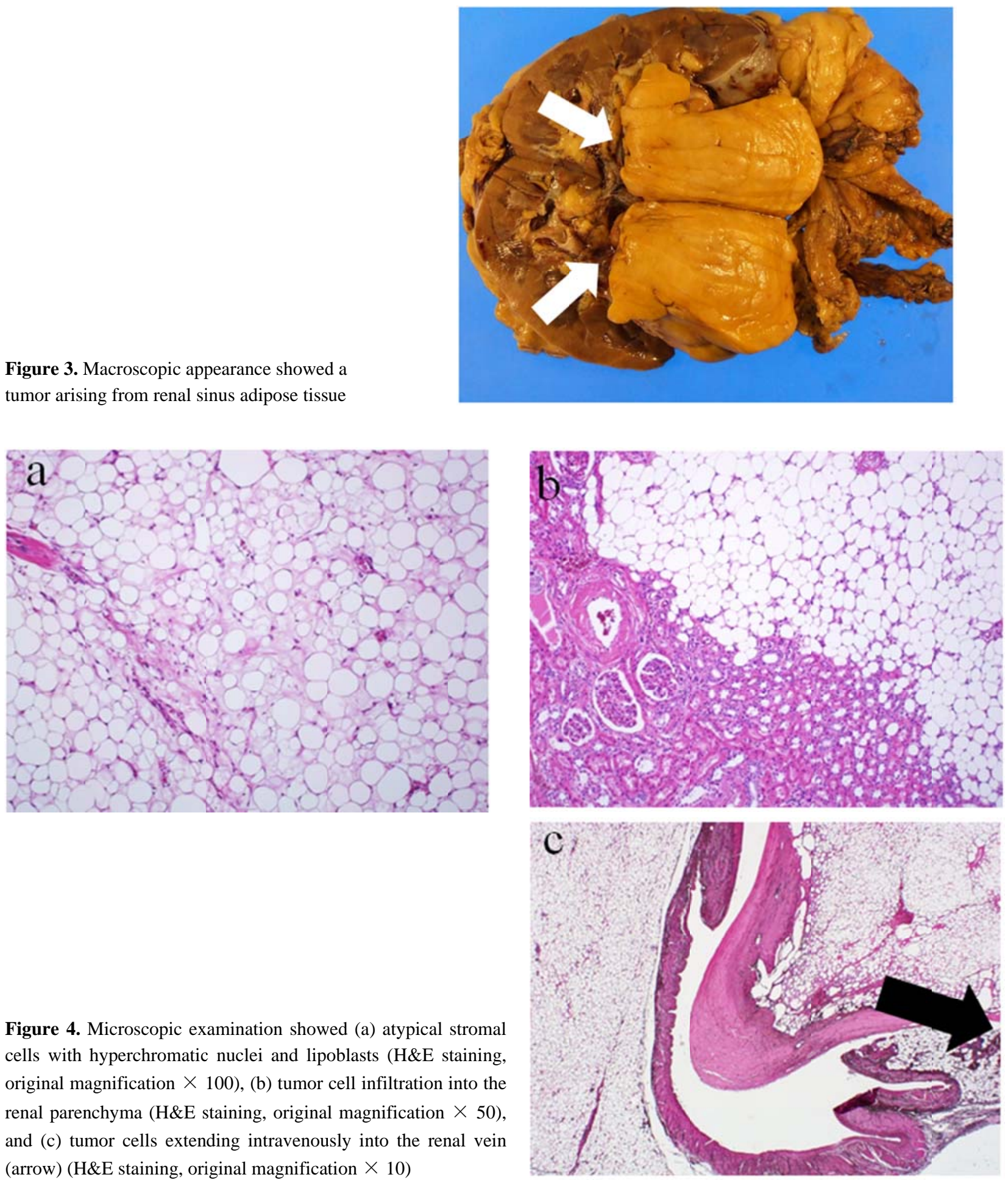

Figure 4. Microscopic examination showed (a) atypical stromal cells with hyperchromatic nuclei and lipoblasts (H\&E staining, original magnification $\times 100$ ), (b) tumor cell infiltration into the renal parenchyma (H\&E staining, original magnification $\times 50$ ), and (c) tumor cells extending intravenously into the renal vein (arrow) (H\&E staining, original magnification $\times 10$ )

\section{Discussion}

The World Health Organization (WHO) divides liposarcoma into four subtypes: atypical lipomatous tumor/well differentiated liposarcoma, dedifferentiated, myxoid, and pleomorphic ${ }^{[3]}$. Identification of lipoblasts is necessary for diagnosing liposarcoma. Criteria for identifying diagnostic lipoblasts include the following: a hyperchromatic indented or sharply scalloped nucleus, existence of lipid-rich droplets in the cytoplasm, and an appropriate histologic background 
(lipomas with fat necrosis, hibernomatous change in lipomas, and fixation artifacts that to some extent resemble lipoblasts) ${ }^{[4]}$. In the present case, lipoblasts were identified in the fat tissue of the renal sinus but not in the perirenal fat. This fact suggests that the tumor arose from the renal sinus.

Renal liposarcoma with intravenous involvement is distinctly rare. There are, to our knowledge, only two published case

reports ${ }^{[5,6]}$, both of which were of 58-year-old women. The first case was of a tumor of the renal sinus extending from the right kidney to the right renal vein. The second case was of a tumor of the renal capsule extending from the left kidney to the IVC. In both cases, radical nephrectomy and excision of the retroperitoneal mass were performed. Neither patient had recurrence of tumor during follow-up periods of 6 months and 24 months, respectively. It was not mentioned in either case whether the tumor had directly invaded into the renal vein or IVC. Although we did not analyze the tumor specimen with thin serial sections in the present patient, we could not find direct invasion into the renal vein or IVC, indicating that the tumor may have extended into the renal vein via the renal parenchyma.

Retroperitoneal liposarcomas have a 20\%-85\% rate of local recurrence after surgical resection. The incidence of recurrence depends on the adequacy of excision ${ }^{[7]}$. Early detection of retroperitoneal liposarcomas is difficult because the symptoms of these tumors appear late and are nonspecific. Retroperitoneal liposarcomas grow slowly in the retroperitoneal space and reach a considerable size before being diagnosed ${ }^{[8]}$.

In the report by Perez et al., a recent meta-analysis of the pertinent literature regarding retroperitoneal soft tissue sarcomas failed to show significant radiotherapy-related survival benefits in the treatment retroperitoneal liposarcoma ${ }^{[9]}$. Matsushita et al reported that chemotherapeutic agents such as doxycycline and ifosfamide demonstrating activity against metastatic sarcoma, but such chemotherapeutic agents have not shown survival benefit ${ }^{[5]}$. Complete resection is crucial regardless of tumor size or adjacent organ involvement in the treatment retroperitoneal liposarcoma because well-differentiated liposarcoma has no indication of radiotherapy and/or chemotherapy ${ }^{[10]}$.

\section{Conclusion}

Liposarcoma arising from renal sinus with intravenous involvement is distinctly rare. Complete resection is recommended in the treatment of retroperitoneal liposarcoma with intravenous involvement.

\section{References}

[1] Srinivas V, Sogani PC, Hajdu SI, et al. Sarcomas of the kidney. J Urol. 1984; 132: 13-16. PMid:6328050.

[2] Novick AC, Campbell SC. Renal tumors. In: Walsh PC, Retik AB, Vaughan ED et al (eds) Campbell's Urology, 8th edn. Saunders, Philadelphia; 2002. p. 2673-2731.

[3] Fletcher CDM, Unni KK, Mertens F. World Health Organization Classification of Tumours. Pathology and Genetics of Tumours of Soft Tissue and Bone. LARC Press, Lyon; 2002. p. 35-46. PMid:11756767.

[4] Weiss SW, Goldblum JR. Enzinger and Weiss's Soft Tissue Tumors, 5th Edition. Mosby Elsevier, Philadelphia; 2008. p. 477-516.

[5] Matsushita M, Ito A, Ishidoya S, et al. Intravenous extended liposarcoma arising from renal sinus. Int J Urol. 2007; 14: 769-770. PMid:17681072. http://dx.doi.org/10.1111/j.1442-2042.2007.01817.x

[6] Ciccarello G, Mucciardi G, Morgia G, et al. A case of renal capsular liposarcoma with intracaval fat thrombus. Eur Urol. 2010; 57: 350-353. PMid:19660855. http://dx.doi.org/10.1016/j.eururo.2009.07.021

[7] Blinder SC, Katz B, Sheridan MF. Retroperitoneal liposarcoma. Ann Surg. 1978; 187: 257-261. http://dx.doi.org/10.1097/00000658-197803000-00008

[8] Seo IY, Won HS, Kim JS, et al. A case of recurrent liposarcoma in retroperitoneum. Korean J Urol. 1994; 35: $1375-78$.

[9] Perez EA, Gutierrez JC, Moffat FL Jr, et al. Retroperitoneal and truncal sarcomas: prognosis depends upon type not location. Ann Surg Oncol. 2007; 14: 1114-1122. PMid:17206483. http://dx.doi.org/10.1245/s10434-006-9255-X

[10] Han HH, Choi KH, Kim DS, et al. Retroperitoneal giant liposarcoma. Korean J Urol. 2010; 51: 579-582. PMid:20733966. http://dx.doi.org/10.4111/kju.2010.51.8.579 